\title{
Caracterização morfológica e molecular de cistos de Giardia duodenalis em amostras clínicas de crianças provenientes de Parnaíba, Piauí
}

\author{
Morphological and molecular characterization of Giardia duodenalis cysts in clinical samples of \\ children from Parnaíba, Piauí \\ Caracterización morfológica y molecular de quistes de Giardia duodenalis en muestras clínicas de \\ niños de Parnaíba, Piauí
}

Edvaldo Pereira de Moura Filho

ORCID: https://orcid.org/0000-0002-3379-9838 Instituto de Educação Superior do Vale do Parnaíba, Brasil E-mail: edvaldopmourafilho@gmail.com

Igor Matheus Oliveira da Silva ORCID: https://orcid.org/0000-0002-8627-4015 Instituto de Educação Superior do Vale do Parnaíba, Brasil E-mail: igor_matheussilva@outlook.com

Gabriel Lucena Cangussu

ORCID: https://orcid.org/0000-0003-2484-0154 Instituto de Educação Superior do Vale do Parnaíba, Brasil E-mail: dr.gabriel.cangussu@gmail.com

Úrsulo Coragem Alves de Oliveira ORCID: https://orcid.org/0000-0003-2496-3402 Instituto de Educação Superior do Vale do Parnaíba, Brasil E-mail: ursulocoragem@gmail.com

João Cordeiro de Andrade Neto ORCID: https://orcid.org/0000-0002-0947-8540 Instituto de Educação Superior do Vale do Parnaíba, Brasil E-mail: joaocordeiro50@gmail.com

José Fernandes Neto

ORCID: https://orcid.org/0000-0002-4931-0988 Universidade de Fortaleza, Brasil E-mail: jfernandesneto@edu.unifor.br

Lucas Oliveira Lemos

ORCID: https://orcid.org/0000-0003-1491-4148 Universidade de Fortaleza, Brasil E-mail: lucasoliv228@edu.unifor.br

Deborah Marinho Paiva Nogueira ORCID: https://orcid.org/0000-0002-7093-148X Universidade de Fortaleza, Brasil

E-mail: deborahmpaivan@edu.unifor.br

Matteus Augusto Oliveira do Amarante de Paulo ORCID: https://orcid.org/0000-0001-9188-9420 Universidade de Fortaleza, Brasil E-mail: matteusaugust@hotmail.com

Vanessa Menezes de Brito Campelo ORCID: https://orcid.org/0000-0002-9469-9857 Instituto de Educação Superior do Vale do Parnaíba, Brasil E-mail: vanessamenesesbrito@gmail.com

\begin{abstract}
Resumo
Atualmente, apesar dos inúmeros avanços científicos e tecnológicos na área de diagnósticos parasitológicos e no campo da saúde coletiva, as parasitoses intestinais ainda constituem um grave problema de Saúde Pública. Possuem distribuição cosmopolita, sendo altamente prevalentes tanto em países em desenvolvimento como em países desenvolvidos. Dentre essas infecções parasitárias, a giardíase, causada pelo protozoário Giardia duodenalis, é considerada a mais comum em humanos. Seu diagnóstico é geralmente realizado através da microscopia convencional, no entanto, dada a sua importância atual métodos moleculares têm sido desenvolvidos para a sua detecção. O objetivo desse trabalho é caracterizar morfológica e molecularmente amostras clínicas de crianças positivas para Giardia duodenalis no município de Parnaíba, Piauí. As amostras fecais foram processadas segundo o
\end{abstract}


método de Faust, onde as positivas foram purificadas para posterior caracterização molecular. Em seguida, foi realizado a extração do material genético e realizada a PCR convencional, através da amplificação de segmentos dos marcadores bg e tpi. Posteriormente, foi elaborado o sequenciamento dos isolados, para identificação das assemblages.Foram analisados um total de 15 casos confirmados de giardíase em habitantes do município de Parnaíba-PI, dos quais, todos foram estratificados e caracterizados como pertencentes ao assemblage A, em que 13 casos $(86,6 \%)$ relataram sintomas diarréicos e nove participantes $(60 \%)$ referiram perda de peso. Ainda são necessários estudos que aprofundem o conhecimento acerca das enteroparasitoses, visto que há carência de mais informações sobre o patógeno e grande parte da população é pouco esclarecida no tocante a transmissão e sintomatologia destas, o que corrobora com os dados epidemiológicos.

Palavras-chave: Enteroparasitose; Giardia duodenalis; PCR; Assemblages; Genótipos.

\begin{abstract}
Currently, despite numerous scientific and technological advances in the area of parasitological diagnoses and in the field of collective health, intestinal parasittosis is still a serious public health problem. They have cosmopolitan distribution, being highly prevalent both in developing and developed countries. Among these parasitic infections, giardiasis, caused by the protozoan Giardia duodenalis, is considered the most common in humans. Its diagnosis is usually carried out through conventional microscopy, however, given its current importance molecular methods have been developed for its detection. The objective of this work is to characterize morphologically and molecularly clinical samples of children positive for Giardia duodenalis in the municipality of Parnaíba, Piauí. Fecal samples were processed according to faust's method, where positive samples were purified for further molecular characterization. Then, the genetic material was extraction and conventional PCR was performed, through the amplification of segments of the bg and tpi markers. Subsequently, the sequencing of the isolates was elaborated to identify the assemblages. A total of 15 confirmed cases of giardiasis were analyzed in inhabitants of the municipality of ParnaíbaPI, all of which were stratified and characterized as belonging to assemblage A, in which 13 cases (86.6\%) reported diarrheal symptoms and nine participants (60\%) reported weight loss. Studies are still needed to deepen the knowledge about enteroparasitosis, since there is a lack of more information about the pathogen and a large part of the population is poorly understood regarding the transmission and symptomatology of these, which corroborates the epidemiological data.
\end{abstract}

Keywords: Enteroparasitosis; Giardia duodenalis; PCR; Assemblages; Genotypes.

\title{
Resumen
}

Actualmente, a pesar de los numerosos avances científicos y tecnológicos en el área de los diagnósticos parasitológicos y en el campo de la salud colectiva, la parasittosis intestinal sigue siendo un grave problema de salud pública. Tienen una distribución cosmopolita, siendo muy frecuentes tanto en los países en desarrollo como en los desarrollados. Entre estas infecciones parasitarias, la giardiasis, causada por el protozoo Giardia duodenalis, se considera la más común en humanos. Su diagnóstico se suele realizar a través de microscopía convencional, sin embargo, dada su importancia actual se han desarrollado métodos moleculares para su detección. El objetivo de este trabajo es caracterizar morfológica y molecularmente muestras clínicas de niños positivos para Giardia duodenalis en el municipio de Parnaíba, Piauí. Las muestras fecales se procesaron de acuerdo con el método de Fausto, donde las muestras positivas se purificaron para una mayor caracterización molecular. Luego, se extracción del material genético y se realizó PCR convencional, mediante la amplificación de segmentos de los marcadores bg y tpi. Posteriormente, se elaboró la secuenciación de los aislados para identificar los ensamblajes. Se analizaron un total de 15 casos confirmados de giardiasis en habitantes del municipio de Parnaíba-PI, todos los cuales fueron estratificados y caracterizados como pertenecientes al conjunto A, en los cuales 13 casos $(86,6 \%)$ reportaron síntomas diarreicos y nueve participantes $(60 \%)$ reportaron pérdida de peso. Aún se necesitan estudios para profundizar en el conocimiento sobre la enteroparasitosis, ya que falta más información sobre el patógeno y gran parte de la poblaciónes poco conocida respecto a la transmisión y sintomatología de estas, lo que corrobora los datos epidemiológicos.

Palabras clave: Enteroparasitosis; Giardia duodenalis; PCR; Assemblages; Genotypes.

\section{Introdução}

A ocorrência de parasitoses intestinais é um grave problema de saúde pública com distribuição global. No Brasil, tal problema está relacionado com a falta de saneamento (Nunes et al., 2018). Estima-se que a prevalência seja entre 2 a 36\% na população geral e alcança até $70 \%$ em crianças no período escolar (Teixeira et al., 2020). Embora as doenças parasitárias estejam mais associadas a baixos níveis socioeconômicos, países em desenvolvimento, como o Brasil, apresentam uma discrepância na situação social e portanto, as altas taxas de infecções por parasitoses não se restringem às regiões menos 
favorecidas (Viana et al, 2017), sendo bastante frequente tanto em ambientes urbanos quanto em rurais, mostrando a importância da melhoria do saneamento e da qualidade de vida em ambos locais (Teixeira et al., 2020; Maia \& Hassum, 2016)

Tais dados podem ser evidenciados por meio da análise das regiões dos biomas da Amazônia, Cerrado e Caatinga, os quais foram observados maior prevalência do assemblage B e A nos dois últimos, respectivamente (Nunes et al., 2018). Embora a justificativa do predomínio do assemblage B na Amazônia se deve por sua rede sanitária deficitária, as regiões Nordeste, contida no bioma Caatinga, apresentam o pior saneamento, e, apesar disso, constitui uma região predominantemente semiárida, de modo que contribui com o aparecimento do assemblage A (Nunes et al., 2018).

A espécie Giardia duodenalis é composta por oito grupos geneticamente distintos (A - H), denominados de assemblages, mas morfologicamente idênticos. A maioria das assemblages parece possuir hospedeiros específicos, como cães, gatos e primatas, gado, cavalo e porcos, contudo somente as assemblages A e B são consideradas oficialmente zoonóticas. (Tungtrongchitr et. al., 2010; Durigan et al., 2014). No entanto, alguns estudos já investigam a possibilidade do assemblage E possuir potencial zoonótico (Fantinatti et al., 2016).

No estado do Piauí, assim como em outros estados, o protozoário patogênico intestinal mais prevalente, principalmente em crianças, é Giardia duodenalis, que acomete em geral 25,6\% da população (Viana et al, 2017; Nunes et al., 2018).). Na cidade de Parnaíba, foi relatada a presença deste protozoário em crianças (Melo et al., 2014), adultos e idosos (Furtado, 2011) em taxas de 29,9\% (Melo et al., 2014, 3,7\% e 11,7\% (Furtado, 2011), respectivamente. Tais parasitas podem causar, em casos crônicos, desnutrição, devido à uma má-absorção intestinal, síndromes diarréicas crônicas e provocando, entre outros sintomas, retardo no crescimento em crianças (Corrêa et al., 2020). Essa doença costuma ser de difícil controle, pois, no tocante ao tratamento, não depende apenas da interferência médica, mas de modificações ambientais e sociais, (Viana et al, 2017; Bacelar et al., 2018) e, por conta da carência sanitária em algumas regiões, contribui com a reemergência (Robertson et al., 2010).

A transmissão em humanos pode ocorrer por via direta, ou seja, de pessoa para pessoa, ou via indireta, por meio da ingestão de água, verduras ou frutas cruas contaminadas pelos cistos de G. duodenalis. Infecções de via indireta, principalmente pela ingestão dos cistos contaminados na água, é a principal rota de transmissão (Dixon, 2021).

Seu diagnóstico é comumente realizado por meio de exame coprológico direto, preferencialmente pela pesquisa de cistos ou trofozoítos do parasito em amostras de fezes coletadas (Leung et al., 2019), sendo considerado o teste padrão-ouro. Contudo, a sensibilidade do método convencional é inferior em relação aos demais métodos detectores de antígenos, pois a identificação da espécie ocorre apenas por meio da observação dos aspectos morfológicos (Minetti et al., 2016). Demais exames diagnósticos compreendem métodos como, hematoxilina férrica, método imunoenzimático qualitativo para a detecção de antígeno específico, específico para G. duodenalis e método de concentração de Faust (Machado et al., 2001)

É pertinente destacar que a diferenciação entre os genótipos da G. duodenalis ocorreu a partir do estudo da mobilidade eletroforética dos cromossomos (Thompson, 2004). Em que houve ampliação e confirmação dessa heterogeneidade com a Reação em Cadeia da Polimerase (PCR) do DNA de G. duodenalis, análise do polimorfismo do comprimento do fragmento de restrição (RFLP) e sequenciamento dos produtos de PCR resultantes. Nesse estudo, observa-se os genes que codificam a $\beta$-giardina (bg), triose fosfato isomerase (tpi), a pequena subunidade do RNA ribossômico (ssu) e glutamato desidrogenase (gdh), em que alguns são mais específicos de certo genótipo (Heyworth et al., 2016).

Ademais, a caracterização molecular através de análises de DNA possibilita confirmar a heterogeneidade deste parasito, servindo como base para um maior esclarecimento a respeito da sua taxonomia (Durigan et al., 2014, Durigan et al., 2017). e do seu ciclo zoonótico de transmissão entre as suas classificações, facilitando distinguir qual genótipo está associado ao quadro de manifestações clínicas e, através das fontes de contaminação e transmissão, buscar medidas profiláticas (Fantinatti et al., 2020). Apesar de existirem inúmeras áreas no Brasil com alta prevalência de G. duodenalis, os estudos de 
genotipagem são relativamente recentes, em decorrência disso, o direcionamento para medidas de controle específicas é dificultado (Durigan et al., 2014; Fantinatti et al., 2020). Portanto, o objetivo desse trabalho é caracterizar morfológica e molecularmente amostras clínicas de crianças positivas para Giardia duodenalis no município de Parnaíba, Piauí.

\section{Metodologia}

Todos os parâmetros éticos foram seguidos com a utilização de Termos de Assentimento Livre e Esclarecido (TALE), com o número de aprovação do comitê de ética 0014.0.045.000-09. A pesquisa foi realizada no município de Parnaíba-PI, situado no extremo norte do Piauí. As amostras de habitantes trabalhadores e crianças matriculadas regularmente em uma creche de zona rural e uma de zona urbana escolhidas aleatoriamente, foram coletadas, com demais indivíduos sendo excluídos, compreendendo assim um conjunto amostral representativo. O método de Faust, que consiste na centrifugoflutuação em sulfato de zinco, foi utilizado no processamento das amostras, com identificação feita em microscópio óptico em aumento de 400x. Uma posterior caracterização molecular foi realizada em resultados positivos. Os cromatogramas das sequências obtidas foram alinhados com o auxílio do programa BioEdit Sequence Alignment Editor versão 7.0, tomando como base sequências homólogas disponíveis no GenBank. A análise filogenética foi realizada usando o método de Neighbor-Joining (NJ) implementada no programa MEGA 5 (Tamura et al., 2011). Os genótipos obtidos foram analisados segundo o método Kappa para análise de concordância entre os dois marcadores utilizados. Os dados obtidos foram analisados pelo programa EPI INFO. Além disso, foi solicitado aos participantes o preenchimento de um formulário em que distinguia idade, sexo e a presença de sintomas diarréicos e perda ponderal.

\section{Resultados e Discussão}

\subsection{Perfil das amostras positivas}

Foram analisados um total de 15 casos confirmados de giardíase em habitantes de Parnaíba-PI, dos quais, todos foram caracterizados como pertencentes ao assemblage A, em que 13 casos $(86,6 \%)$ relataram sintomas diarréicos e nove participantes $(60 \%)$ referiram perda de peso. 
Tabela 1 - Variáveis analisada pelo Teste Exato de Fisher para comparação entre duas proporções.

\begin{tabular}{|c|c|c|c|}
\hline VARIÁVEIS & $\mathbf{N}^{\mathbf{o}}$ & $\%$ & P-VALOR \\
\hline \multicolumn{4}{|l|}{ Faixa etária } \\
\hline 4-56 anos & 15 & $100 \%$ & 0,512 \\
\hline \multicolumn{4}{|l|}{ Sexo } \\
\hline Feminino & 7 & $46,60 \%$ & 0,651 \\
\hline Masculino & 8 & $53,30 \%$ & \\
\hline \multicolumn{4}{|l|}{ Diarreia } \\
\hline Sim & 13 & $86,60 \%$ & 0,5 \\
\hline Não & 2 & $13,30 \%$ & \\
\hline \multicolumn{4}{|l|}{ Perda de peso } \\
\hline Sim & 9 & $60 \%$ & 0,681 \\
\hline Não & 6 & $40 \%$ & \\
\hline
\end{tabular}

\subsection{Amplificação e Sequenciamento}

A amplificação pelo teste PCR obteve resultados positivos em seis casos (40\%) para o gene by e cinco casos (33,3\%) para o gene tpi. Em que, cinco amostras do gene bg (83,3\%) foram classificadas como assemblage A e um caso (16,6\%) como assemblage misto $\mathrm{A} / \mathrm{B}$.

Tabela 2 - Estratificação das Amostras.

\begin{tabular}{ccccccc}
\hline ISOLADO & IDADE & SEXO & bg & tpi & ASSEMBLAGE & SUB-ASSEMBLAGE \\
\hline PV01 & 4 & M & + & + & A/B & AII \\
PV02 & 7 & F & + & + & A & AII \\
PV03 & 14 & F & + & - & A & AII \\
PV04 & 56 & M & + & + & A & AII \\
PV05 & 35 & F & + & + & A & AII \\
PV06 & 18 & F & + & + & A & AII \\
\hline
\end{tabular}

Fonte: Pereira et al. (2021).

\subsection{Relação Inter-assemblage com genes bg e tpi}

Foram analisadas as principais manifestações clínicas e consistência das fezes em amostras concordantes interassemblage e em genes bg e tpi. Com quatro casos (80\%) apresentando sintomas diarréicos, dois (40\%) manifestando perda de peso e dois participantes $(40 \%)$ referindo dores abdominais. 
Tabela 3. Relação dos isolados com concordância inter-assemblage para os dois marcadores (bg e tpi), associados às principais manifestações clínicas apresentadas e consistência das fezes

\begin{tabular}{cccc}
\hline ISOLADO & ASSEMBLAGE & MANIFESTAÇÕES CLÍNICAS & CONSISTENCIA DAS FEZES \\
\hline PV01 & A & Assintomático & Pastosa \\
PV02 & A & Perda de peso, Diarreia & Pastosa \\
PV04 & A & Diarreia, Perda de peso & Pastosa \\
PV05 & A & Diarreia, Dor abdominal & Pastosa \\
PV06 & A & Diarreia, Dor abdominal
\end{tabular}

Fonte: Pereira et al. (2021).

Estima-se que 200 milhões de pessoas estejam infectadas de forma sintomática nos continentes da África, América Latina e Ásia, com cerca de 500 mil novos casos por ano. No estado do Piauí, a G. duodenalis acomete cerca de 25,6\% da população (Viana et al., 2017). Entre as principais transmissões de doenças a partir dos protozoários por meio da água contaminada, ao redor do mundo, estima-se que 40,6\% estejam relacionadas a $G$. duodenalis, nos países Europeus e da América do Norte (Karanis, Kourenti \& Smith, 2006). Além disso, as gastroenterites, como a Giardíase, estão bastante relacionadas à infecção por meio de veiculação hídrica, ou seja, água não tratada de forma correta, sendo visto como um fator de risco para problemas de saúde (Durigan et al., 2014; Grott et al., 2016).

Dada à importância deste meio de infecção, esforços têm sido realizados para desenvolver técnicas mais efetivas para a detecção das mesmas em amostras ambientais (Baque et al., 2011). Assim, o controle do ambiente é um importante modo de prevenção dessas doenças em humanos (Rocha, 2009).

No Brasil, o Ministério da Saúde, por meio da portaria no 518, de 25 de março de 2004, recomendou a inclusão da pesquisa do gênero Giardia em água para consumo humano, objetivando uma maior atenção das empresas de tratamento de água acerca deste parasito (Robertson et al., 2010). Observa-se assim uma expressiva importância epidemiológica, justificada devido ao seu significativo potencial zoonótico, amplo número de hospedeiros susceptíveis, grande variabilidade genética, fácil disseminação e difícil controle de contaminação ambiental (Soliman, Fuentes \& Rubio, 2011). Diversos animais têm sido considerados como potenciais reservatórios, incluindo os animais domésticos como ruminantes, monogástricos e animais aquáticos (Ryan \& Caccio, 2013), sendo mais frequente em cães e gatos (Soliman, Fuentes \& Rubio, 2011).

Vale salientar que este protozoário é responsável por quadros clínicos que variam desde assintomáticos, diarreia aguda e até diarreias crônicas debilitantes, com esteatorréia e perda de peso, dependendo da suscetibilidade do hospedeiro e qual assemblage de Giardia foi adquirido pelo paciente. Ademais, outros sintomas, como urticária, constipação, infecções gástricas associadas também são relatadas (Furtado et al., 2011). Em 2004 a giardíase foi incluída na lista de doenças negligenciadas da OMS, sendo inserida devido a fatores como a maior relevância epidemiológica em populações financeiramente menos favorecidas, causando uma escassez de investimentos em pesquisas de fármacos atualizados (Savioli et al., 2006).

Ademais, a taxonomia da Giardia duodenalis ainda hoje se encontra em discussão, visto que as características morfológicas de cistos e trofozoítos recuperados de humanos e mamíferos infectados são idênticas. No entanto, avanços nos conhecimentos de biologia molecular e a utilização de técnicas moleculares como PCR, permitiu observar a ampla variedade genética desta espécie e a diferença entre os membros, sendo G. duodenalis, considerada uma espécie geneticamente complexa (Caccio \& Ryan, 2008; FENG \& XIAO, 2011). Além disso, as diferenças quanto à infectividade no hospedeiro, padrões 
metabólicos e de crescimento, podem ser explicadas devido ao alto grau de variabilidade genética entre os isolados de $G$. duodenalis (Wielinga; Thompson, 2007). Demonstrando assim, que essas diferenças estão relacionadas com o curso da infecção e a resposta imune do indivíduo (Caccio \& Ryan, 2008)

A prevalência quanto aos genótipos de Giardia duodenalis pode variar de acordo com o país ou pode variar dentro do próprio país, como no Brasil, devido às suas proporções territoriais. No Brasil, os escassos estudos relacionados à epidemiologia molecular de G. duodenalis em humanos, determinam a assemblage B como prevalente em estados, como Ceará, Minas Gerais e Paraná (Faria et al., 2016; Nunes et al., 2018). No estado do Rio de Janeiro, os estudos divergem sendo visto, em alguns momentos, a prevalência do assemblage A, porém mais recentemente foi verificado a presença do assemblage B (Faria et al, 2016), dessa forma, sendo necessário mais estudos. No entanto, vale ressaltar que foi observado a mudança do perfil genético ao longo dos anos no Brasil, sendo analisado no ano de 2012, o assemblage mais prevalente era o A e, nos anos subsequentes, houve a alteração para o assemblage B nos estados do Brasil (Faria et al., 2016).

Então, entende-se que apesar das informações conhecidas acerca dos genótipos de G. duodenalis ainda são escassas as pesquisas de caracterização molecular deste enteroparasita. Estudos moleculares e de análise de variação genotípica, permitem conhecer o padrão de frequência dos principais genótipos, além de fornecer informações sobre a existência de coinfecções, contribuindo assim, para adoção de medidas eficazes de controle e de diagnóstico, além de facilitar a compreensão quanto à variação clínica da doença (Sprong et. al., 2009. Durigan et al., 2014, Durigan et al., 2017).

\section{Conclusão}

Diante do cenário contemporâneo, ainda são necessários estudos que aprofundem o conhecimento acerca das enteroparasitoses, visto que carece de mais informações sobre o patógeno e grande parte da população é pouco esclarecida no tocante a transmissão e sintomatologia destas, o que corrobora com os dados epidemiológicos. Ademais, este trabalho revela dados pertinentes acerca da prevalência de parasitas em uma amostra no município de Parnaíba-PI, demonstrando que a $G$. duodenalis, caracterizado como pertencente ao assemblage A, foi verificado como mais prevalente na região analisada, sendo verificado que a maioria apresenta sintomas diarreicos, seguido de perda de peso e dor abdominal. Logo, auxilia em estudos posteriores, servindo de base para pesquisas futuras de comparação de dados epidemiológicos deste protozoário.

\section{Referências}

Bacelar, P. A. A., Santos, J. P. dos, Monteiro, K. J. L., Calegar, D. A., Nascimento, E. F. do, \& Costa, F. A. C. (2018). Parasitoses intestinais e fatores associados no estado do Piauí: uma revisão integrativa. Revista Eletrônica Acervo Saúde, 10(4), 1802-1809. https://doi.org/10.25248/reas223_2018

Baque, R. H., Gilliam, A. O., Robles, L. D., Jakubowski, W., \& Slifko, T. R. (2011). A real-time RT-PCR method to detect viable Giardia lamblia cysts in environmental waters. Water research, 45(10), 3175-3184. https://doi.org/10.1016/j.watres.2011.03.032

Cacciò, S. M., \& Ryan, U. (2008). Molecular epidemiology of giardiasis. Molecular and biochemical parasitology, 160(2), 75-80. https://doi.org/10.1016/j.molbiopara.2008.04.006

Corrêa, C., Oliveira-Arbex, A. P., David, É. B., \& Guimarães, S. (2020). Genetic analysis of Giardia duodenalis isolates from children of low-income families living in an economically successful region in Southeastern Brazil. Revista do Instituto de Medicina Tropical de Sao Paulo, 62, e20. https://doi.org/10.1590/S1678-9946202062020

Dixon, B. R. (2021). Giardia duodenalis in humans and animals - Transmission and disease. Research in Veterinary Science, 135(June), 283-289. https://doi.org/10.1016/j.rvsc.2020.09.034

Durigan, M., Abreu, A. G., Zucchi, M. I., Franco, R. M. B., \& De Souza, A. P. (2014). Genetic diversity of giardia duodenalis: Multilocus genotyping reveals zoonotic potential between clinical and environmental sources in a metropolitan region of brazil. PLoS ONE, 9(12), 1-27. https://doi.org/10.1371/journal.pone.0115489

Durigan, M., Ciampi-Guillardi, M., Rodrigues, R. C. A., Greinert-Goulart, J. A., Siqueira-Castro, I. C. V., Leal, D. A. G., Yamashiro, S., Bonatti, T. R., Zucchi, M. I., Franco, R. M. B., \& de Souza, A. P. (2017). Population genetic analysis of Giardia duodenalis: genetic diversity and haplotype sharing between clinical and environmental sources. MicrobiologyOpen, 6(2), 1-11. https://doi.org/10.1002/mbo3.424 
Fantinatti, M., Bello, A. R., Fernandes, O., \& Da-Cruz, A. M. (2016). Identification of Giardia lamblia Assemblage E in Humans Points to a New Anthropozoonotic Cycle. The Journal of infectious diseases, 214(8), 1256-1259. https://doi.org/10.1093/infdis/jiw361

Fantinatti, M., Gonçalves-Pinto, M., Lopes-Oliveira, L. A. P., \& Da-Cruz, A. M. (2020). Epidemiology of giardia duodenalis assemblages in brazil: There is still a long way to go. Memorias Do Instituto Oswaldo Cruz, 115(11), 1-12. https://doi.org/10.1590/0074-02760200431

Faria, C. P., Zanini, G. M., Dias, G. S., da Silva, S., \& Sousa, M. (2016). Molecular Characterization of Giardia lamblia: First Report of Assemblage B in Human Isolates from Rio de Janeiro (Brazil). PloS one, 11(8), e0160762. https://doi.org/10.1371/journal.pone.0160762

Feng, Y., \& Xiao, L. (2011). Zoonotic potential and molecular epidemiology of Giardia species and giardiasis. Clinical microbiology reviews, 24(1), 110-140. https://doi.org/10.1128/CMR.00033-10

Furtado, L. F. V., \& Melo, A. C. F. L. (2011). Prevalence and epidemiological aspects of intestinal parasites in elderly of Parnaíba, State of Piaui. Revista Da Sociedade Brasileira de Medicina Tropical, 44(4), 513-515. https://doi.org/10.1590/S0037-86822011000400023

Grott, S. C., Hartmann, B., Filho, H. H. S., Franco, R. M. B., Goulart, J. A. G. (2016). Detecção de cistos de Giardia spp. e oocistos de Cryptosporidium spp. na água bruta das estações de tratamento no município de Blumenau, SC, Brasil. Ambiente e Agua - An Interdisciplinary Journal Of Applied Science, 11(3), 689-701. http://dx.doi.org/10.4136/ambi-agua.1853.

Heyworth M. F. (2016). Giardia duodenalis genetic assemblages and hosts. Parasite, 23(13), 1-5. https://doi.org/10.1051/parasite/2016013.

Karanis, P., Kourenti, C., \& Smith, H. (2006). Waterborne transmission of protozoan parasites: A worldwide review of outbreaks and lessons learnt. Journal of Water and Health, 5(1), 1-38. https://doi.org/10.2166/wh.2006.002

Leung, A., Leung, A., Wong, A., Sergi, C. M., \& Kam, J. (2019). Giardiasis: An Overview. Recent patents on inflammation \& allergy drug discovery, 13(2), 134-143. https://doi.org/10.2174/1872213X13666190618124901

Machado, R. L., Figueredo, M. C., Frade, A. F., Kudó, M. E., Silva Filho, M. G., \& Póvoa, M. M. (2001). Comparação de quatro métodos laboratoriais para diagnóstico da Giardia lamblia em fezes de crianças residentes em Belém, Pará. Revista Da Sociedade Brasileira de Medicina Tropical, 34(1), 91-93. https://doi.org/10.1590/S0037-86822001000100014

Maia, C. V. A.; Hassum, I. C. (2016). Intestinal Parasites and Social-Sanitary Aspects in Northeastern Brazil in. Hygeia - Revista Brasileira de Geografia Médica e Da Saúde, 12(23), 20-30.

Melo, A. C. F. L., Ceia Junior, E. A. de, Azevedo, I. M. de, Souza, P. D. de A., Miranda, C. R. L., Borges, E. P., \& Trindade, R. A. da. (2014). Aspectos Epidemiológicos das Enteroparasitoses em Crianças de uma Unidade Pública de Ensino de Parnaíba, Piauí. UNOPAR Cient., Ciênc. Biol. Saude, 16(3), 191196. https://doi.org/10.17921/2447-8938.2014v16n3p\%25p

Minetti, C., Chalmers, R. M., Beeching, N. J., Probert, C., \& Lamden, K. (2016). Giardiasis. BMJ (Online), 355(i5369), 1-9. https://doi.org/10.1136/bmj.i5369

Nunes, B. C., Calegar, D. A., Pavan, M. G., Jaeger, L. H., Monteiro, K. J. L., dos Reis, E. R. C., Lima, M. M., Bóia, M. N., \& Carvalho-Costa, F. A. (2018). Genetic diversity of Giardia duodenalis circulating in three Brazilian biomes. Infection, Genetics and Evolution, 59(2017), 107-112. https://doi.org/10.1016/j.meegid.2018.02.001

Robertson, L. J., Hanevik, K., Escobedo, A. A., Mørch, K., \& Langeland, N. (2010). Giardiasis--why do the symptoms sometimes never stop?. Trends in parasitology, 26(2), 75-82. https://doi.org/10.1016/j.pt.2009.11.010

Ryan, U., \& Cacciò, S. M. (2013). Zoonotic potential of Giardia. International journal for parasitology, 43(12-13), 943-956. https://doi.org/10.1016/j.ijpara.2013.06.001

Savioli, L., Smith, H., \& Thompson, A. (2006). Giardia and Cryptosporidium join the "Neglected Diseases Initiative." Trends in Parasitology, 22(5), 203-208. https://doi.org/10.1016/j.pt.2006.02.015

Soliman, R. H., Fuentes, I., \& Rubio, J. M. (2011). Identification of a novel Assemblage B subgenotype and a zoonotic Assemblage C in human isolates of Giardia intestinalis in Egypt. Parasitology international, 60(4), 507-511. https://doi.org/10.1016/j.parint.2011.09.006

Sprong, H., Cacciò, S. M., van der Giessen, J. W., \& ZOOPNET network and partners (2009). Identification of zoonotic genotypes of Giardia duodenalis. PLoS neglected tropical diseases, 3(12), e558. https://doi.org/10.1371/journal.pntd.0000558

Teixeira, P. A., Fantinatti, M., Gonçalves, M. P., \& Silva, J. S. (2020). Parasitoses intestinais e saneamento básico no Brasil: estudo de revisão integrativa. Brazilian Journal of Development, 6(5), 22867-22890. https://doi.org/10.34117/bjdv6n5-006

Thompson, R. C. A. (2004). The zoonotic significance and molecular epidemiology of Giardia and giardiasis. Veterinary Parasitology, 126(1-2), 15-35. https://doi.org/10.1016/j.vetpar.2004.09.008

Tungtrongchitr, A., Sookrung, N., Indrawattana, N., Kwangsi, S., Ongrotchanakun, J., \& Chaicumpa, W. (2010). Giardia intestinalis in Thailand: identification of genotypes. Journal of health, population, and nutrition, 28(1), 42-52. https://doi.org/10.3329/jhpn.v28i1.4522

Viana, M. L., Fialho, N. R., Rocha, S. M. S., Alves, T. C. L. A., Trindade, R. A. da, \& Melo, A. C. F. L. (2017). Parasitoses intestinais e a inter-relação com os aspectos socioeconômicos de indivíduos residentes em um povoado rural (Rosápolis de Parnaíba-PI). Scientia Plena, 13(8), 1-10. https://doi.org/10.14808/sci.plena.2017.086801

Wielinga, C. M., \& Thompson, R. C. (2007). Comparative evaluation of Giardia duodenalis sequence data. Parasitology, 134(12), 1795-1821. https://doi.org/10.1017/S0031182007003071 